\title{
FORMAÇÃO E ACESSIBILIDADE: por uma escola e uma sociedade inclusivas
}

Lucia De Anna

Università degli Studi di Roma "Foro Italico"

Alessio Covelli

Università degli Studi di Roma "Foro Italico"

\begin{abstract}
Resumo
Este artigo discute a formação de inicial e continuada de professores, tendo como referência documentos formulados na Itália desde 1977 e recomendações da União Europeia relativos à educação escolar das pessoas com necessidades educacionais especiais (NEE). O artigo é dividido em quatro partes. Na primeira, introduz-se a discussão das atividades de formação e de pesquisa no contexto de uma escola inclusiva para, em seguida, focalizar os aspectos facilitadores e dificultadores para a promoção da acessibilidade nos ambientes escolares. Na terceira parte são apresentados dados de uma pesquisa realizada com professores participantes de um curso de formação. A partir das intervenções desenvolvidas, buscou-se identificar mudanças no olhar e nas práticas dos docentes em relação aos estudantes em situação de deficiência. Por fim, aborda-se na última parte do texto, a temática da colaboração entre a escola e os parceiros externos, como um dos aspectos centrais e igualmente desafiador na construção de uma escola inclusiva.
\end{abstract}

Palavras-chave: formação de professores; educação inclusiva; pessoas em situação de deficiência; necessidades educacionais especiais; colaboração.

\begin{abstract}
This article discusses pre-service and in-service teacher training on the basis of documents from Italy since 1977 and recommendations of the European Union regarding the education of people with special educational needs (SEN). The article is divided into four parts. Firstly, the discussion about training and research activities is introduced in the context of an inclusive school; secondly, the facilitating and hindering aspects for the promotion of accessibility in school environments are brought to light. Subsequently, data from a survey conducted with teachers participating in a training course is presented. Based on the interventions developed, we sought to identify a change in the teacher's perceptions and practice in relation to students with disabilities. Finally, in the fourth and final part of the text, the theme of collaboration between schools and the external partners is approached, as one of the central and equally challenging aspects in building an inclusive school.
\end{abstract}

Keywords: teacher training; inclusive education; people with disabilities; special educational needs; collaboration. 


\section{Introdução: Formação e pesquisa no contexto de uma escola inclusiva}

Os professores estão preparados para abordar todos os desafios que a sociedade coloca para os jovens na realidade cotidiana? De um lado, eles devem responder às necessidades específicas das pessoas em situação de deficiência, mas há outros problemas educativos como a violência, o fracasso escolar, o abandono, as diferenças e mal-estar social e cultural, as dificuldades para educar os alunos para a vida ativa e para o fato de que enfrentam poucas oportunidades de inserção no mundo do trabalho. Todos estes elementos são cada vez mais evidentes em nossa sociedade atual. À luz destas questões, talvez devamos reinventar a escola para torná-la realmente acessível e inclusiva. Precisamos promover o diálogo entre as práticas e a pesquisa, e verificar se a formação dos professores apoia ou dificulta a exigência de reinvenção da escola. Como ela é concebida e instaurada?

Mas a formação dos professores não basta para resolver os diferentes problemas se a sociedade não for capaz de compreender as necessidades dos jovens e de responder às suas demandas, se não oferecer oportunidades diversificadas de aprendizagem para que eles tenham êxito na escola e na vida. É preciso construir um plano de formação sistemática de todos os atores envolvidos nesse processo. Para tanto, é importante que professores, lideranças e gestores sejam formados para tornar a escola acessível e inclusiva a fim de fornecer múltiplas oportunidades de aprendizagem e favorecer o êxito de cada aluno. $\mathrm{O}$ desenvolvimento de competências diversificadas para o acesso aos saberes é um elemento crucial de uma escola inclusiva e sua implementação deve envolver os professores e responsáveis pelo estabelecimento de ensino com o objetivo de influenciar o desenvolvimento das crianças por meio da educação e assim contribuir positivamente para a sociedade. (OECD, 2019).

Tentaremos explorar, neste artigo, algumas das questões que se encontram em discussão atualmente em nosso país, tendo como referência pesquisas feitas entre os anos de 2000 e 2018 sobre a formação dos professores e, particularmente, sobre os professores especializados. Consideramos, para tanto, a Recomendação europeia de 2006 (revisada em 2018) acerca das oito competências-chave ${ }^{1}$, em conexão com o Decreto Ministerial de 3009-2011, do Ministro da Educação da Itália (DE ANNA, 2016a; 2018). Atentamo-nos, ainda, para aspectos preliminares de grande importância para a construção de competências:

1. As representações dos professores e suas ressonâncias para as práticas educativas e pedagógicas.

2. As motivações para a formação e suas expectativas.

3. A importância da organização da escola e da interação entre os diversos parceiros para os processos de inclusão.

O objetivo de nossas pesquisas é compreender sob quais condições e por meio de quais instrumentos a escola pode-se constituir, para todas as crianças, inclusive para as mais frágeis, e considerando a singularidade de cada uma delas, uma oportunidade de se construir e de tecer sua vida de adulto e de cidadão em uma sociedade plural. Como os professores representam as crianças com necessidades especiais (NEE) e como interagem 
com elas? Para além das leis, dos textos, das declarações, essas são questões essenciais, que devem ser observadas. A complexidade do movimento inclusivo ultrapassa as fronteiras geográficas, confrontando-se com a realidade de diferentes países, e deve examinar as especificidades históricas, culturais, políticas e/ou econômicas de cada território. Um outro ponto a ser observado diz respeito a como considerar, nas práticas educativas, os seguintes elementos que experimentamos e exprimimos:

- Elaborar projetos e planejar o programa escolar (Lei 517/77) tendo em vista a criação de contextos acessíveis para a inclusão (DE ANNA, 2014a);

- Oferecer uma pluralidade de oportunidades na perspectiva de estabelecer um projeto de vida que compreenda as disciplinas e programas da escola de forma pluri e interdisciplinar.

- Colaborar e participar, contando com o envolvimento de todos os professores - das disciplinas e de apoio: o diretor, equipe de suporte, família, dente outros atores.

- Considerar que atividades realizadas em classe são para todos, sem exceção. Os estudantes podem aprender por meios distintos na sala de aula, e os professores podem introduzir estratégias didáticas que garantam a apresendizem de todos em função das inteligências múltiplas (SCHOVANEC, 2018) e da "especial normalidade" (IANES, 2006).

- A aprendizagem cooperativa representa uma das mais eficazes metodologias ou estratégias didáticas, por ser acolhedora e relacional, favorecendo o êxito na condução da classe em um sentido inclusivo e possibilitando o desenvolvimento de competências por parte de todos os alunos.

- Favorecer a autodeterminação e a participação ativa da pessoa como possibilidade de que ela realize suas escolhas e aspirações tendo em vista o desenvolvimento de seu projeto de vida (EBERSOLD, 2018).

As práticas educativas devem permitir a cada aluno exercer seu papel e vivenciar a experiência desafiadora da educação por meio das formas possíveis, dos estilos de aprendizagem e das possibilidades de aprendizagem colocadas para todos e para cada um na escola.

Os alunos em situação de deficiência, com necessidades especiais ou distúrbios de aprendizagem (WARNOCK REPORT, 1978), devem sentir, escutar, perceber que eles têm a possibilidade de terem êxito em um contexto acolhedor e não discriminatório. Que eles têm o direito a um lugar em uma escola que é para todos, onde eles não precisam pedir para entrar ou terem a expectativa se serão admitidos e acolhidos. Isso deve ser estendido às diferentes dimensões existenciais no âmago da sociedade (DE ANNA 1998; 2014; 2016a; 2018; GARDOU, 2012).

Estes valores fundamentam a concepção de inclusão e as atividades de pesquisa e de formação dos cursos de Pedagogia inclusiva ministrados no âmbito da formação inicial de professores, em serviço e continuada, que são coordenados pelo grupo de pesquisa da Universidade de Roma 'Foro itálico'. 
A partir desses apontamentos, passaremos a apresentar os temas que serão discutidos nos próximos tópicos do artigo.

Na segunda seção nós descrevemos os obstáculos e os facilitadores que atuam na instauração dos princípios necessários para a promoção da acessibilidade nos ambientes escolares. Nas pesquisas realizadas sobre as representações sociais da deficiência (DE ANNA; COVELLI, 2013; COVELLI, 2015; 2016a; 2016b) é demonstrado que a mudança de olhar para as diversidades humanas, incluindo as situações de deficiência, é o primeiro ponto propulsor para o desenvolvimento de uma cultura inclusiva e para a promoção da acessibilidade na sociedade, em todas as dimensões existências de vida. Este processo não pode ignorar o papel e a função educativa da escola e, consequentemente, a importância da cultura na representação dos professores, que devem convergir a sua visão em direção à acessibilidade de todos e a um movimento de ultrapassar os estereótipos e preconceitos.

$\mathrm{Na}$ terceira seção, descrevemos o processo de análise e as metodologias utilizadas em uma pesquisa que visava compreender as motivações, as percepções e as observações expressas pelos professores Abillitati, quer dizer, pelos professores que possuem habilitação para ensinar nos diferentes níveis de ensino, seja na escola maternal, primária, secundaria I e II, e que atuaram no ano acadêmico 2012-2013 e nas SSIS 1 (ano acadêmico 2009). A maior parte dos professores já exerciam a função de professor e muitos trabalhavam como professores de apoio, mas em situação precária e sem o título de especialização. A pesquisa envolveu 240 docentes que foram separados em 48 grupos focais. Os dados levantados nesta intervenção foram comparados, posteriormente, com dados de uma outra investigação, realizada com alunos de anos anteriores, e que envolvia uma amostra de 300 docentes. A pesquisa teve continuidade com outras amostragens e outros grupos de discussão.

Uma primeira fase da pesquisa foi publicada como conclusão de um projeto financiado pela Universidade de Roma Foro itálico, no livro Teaching Accessibility and inclusion (DE ANNA, 2016a), em particular no capítulo 9, e também no capitulo 10, escrito com Covelli (2016b). Essas publicações sintetizam as considerações apresentadas pelos professores sobre as transformações obtidas durante as ações de formação, as atividades realizadas nos estágios e nas práticas educativas.

Na quarta seção, abordamos o tema da colaboração entre escola e parceiros externos, como organizações locais, serviços sociais e de saúde, e estabelecemos uma confrontação desses dados com as primeiras pesquisas realizadas entre os anos de 1992 e 1999 sobre os indicadores de qualidade da OCDE (1999).

\section{Educar para o reconhecimento da diversidade: representações sociais e cultura inclusiva}

A mudança de percepção em torno do desenvolvimento de uma cultura inclusiva se origina da função educativa e formadora da escola (COVELLI, 2016a; 2016 b). A escola como mediadora entre alunos, sociedade e cultura (MOLITERNI, 2013) pode favorecer o 
encontro entre as diferenças humanas e promover o conhecimento recíproco entre pessoas diferentes. Diante disso, quais novas formas de representação deveríamos privilegiar na literatura, na sociedade, na escola, nas mídias? Como contribuir para a promoção de uma sociedade e uma educação inclusivas? Em direção a qual perspectiva de representação social nós devemos nos orientar para tornar o ambiente escolar acessível e disponibilizar ferramentas para a construção de um projeto de vida dos alunos em situação de deficiência e com necessidades especiais?

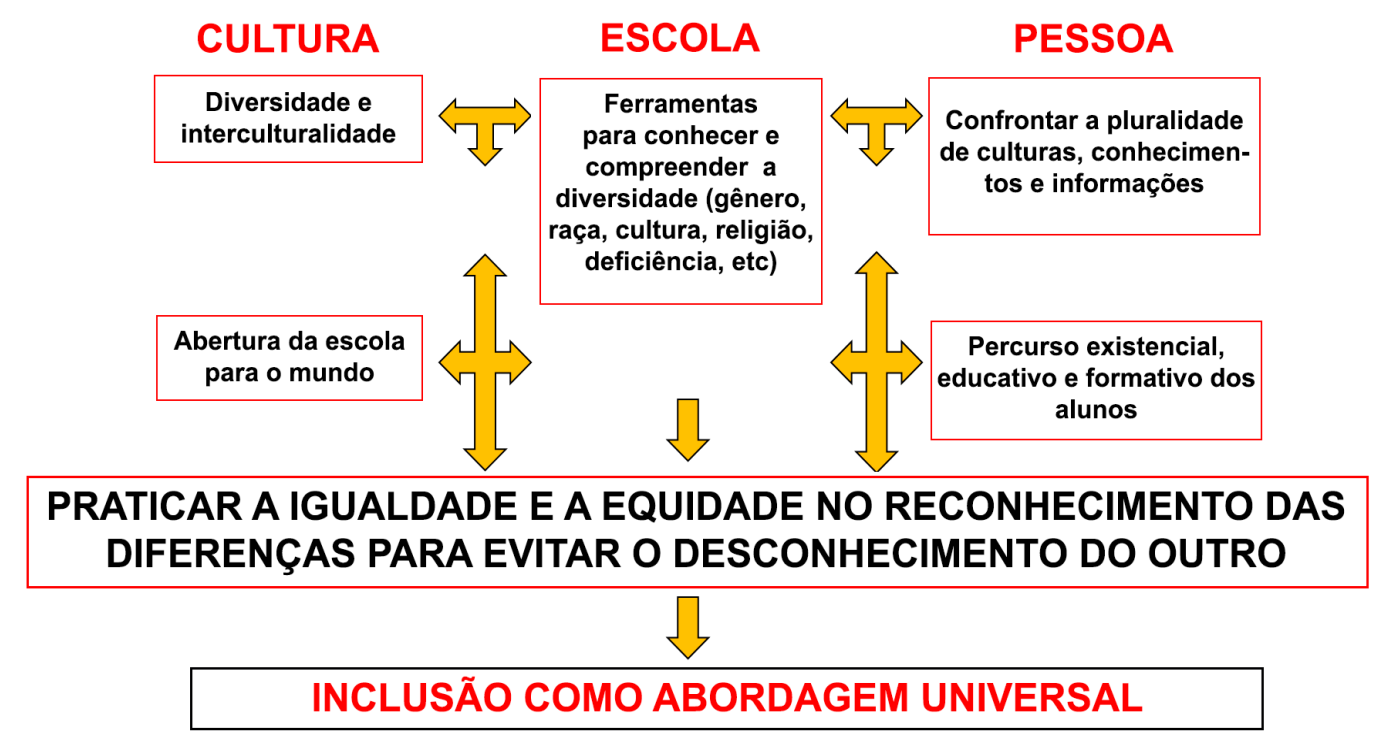

As análises da literatura e os estudos conduzidos nos últimos anos sobre as representações sociais das pessoas em situações de deficiência, dos grupos sociais minoritários e mais frágeis evidenciam a constituição de uma perspectiva questionável, pois a utilização de estereótipos e preconceitos se baseia em uma concepção de oposição cultural e de exclusão mútua entre os conceitos de normalidade e de diversidade (COVELLI, 2015; 2016a; 2016b; DE ANNA, 2016a; 2016b)

A deficiência e o reconhecimento das diferenças em geral exprimem a contradição inerente à relação entre esses dois conceitos porque, apesar das instâncias de reconhecimentos da normalidade demarcarem as situações consideradas fora do comum, afirmam, ao mesmo tempo, sua diversidade e sua normalidade. É necessário, portanto, ir além de uma visão de exclusão mútua entre esses dois valores para conduzir a diversidade rumo a uma perspectiva de normalidade (IANES, 2006) que não seja um achatamento da complexidade e da especificidade da pessoa e de seus contextos de vida, mas sim uma declaração de equidade.

Nesse sentido, não podemos construir uma verdadeira sociedade inclusiva se a escola não educar para o reconhecimento da diferença como valor e condição antológica do ser humano (DE ANNA; GASPARI; MURA, 2015; COVELLI, 2016a). Esta ação deveria ser 
iniciada pelo Estado e por suas instituições, tendo um olhar particular para os professores e para os formadores, pois se trata de uma questão que vai além da sensibilização dos atores. Ela remete, retomando a expressão de Freire (1973; 2004), à conscientização das pessoas para que uma real mudança sociocultural se concretize. A formação dos professores em todos os níveis se funda nesse postulado e considera as pessoas em situação de deficiência ou com necessidades especiais como cidadãos, assim como os outros.

Os princípios constitucionais, as leis e as linhas guias do Ministério da Educação italiano afirmam este princípio: O reconhecimento dos direitos de cidadania como uma condição preliminar para a realização dos processos de inclusão e de emancipação. Os alunos em situação de deficiência não são portadores de deficiência como no modelo tradicional de cuidados e de assistência. Eles são portadores de direitos que determinam o princípio da equidade e um reconhecimento apropriado (HONNETH, 2002), fundado no pluralismo cultural (FERRUCCI, 2004) e no pluralismo identitário (REMOTTI, 2011), que têm como base uma diversidade de representações e de reconhecimento que tocam diferentes dimensões e experiências de vida das pessoas em situações de deficiência ou com necessidades especiais. Nesse sentido, os processos de educação inclusiva necessitam de uma transformação dos contextos em termos das políticas, das práticas e das culturas (AINSCOW, 2014). "A inclusão e a equidade são os princípios gerais que guiam todas as políticas, programas e práticas educativas"' (UNESCO, 2017, p. 17).

Na Itália, a mudança de perspectiva para uma visão inclusiva concretizou-se pela reforma da escola em 1974, com a leis 517-1977 e 104-1992. O legislador favoreceu uma mudança do contexto escolar para reduzir as diferenças sociais e permitir a todos (incluindo as pessoas em situação de deficiência) de frequentar uma escola que fosse para todos. $\mathrm{O}$ legislador imaginou, como prioridade, um novo contexto no qual não se objetivava apenas abrir as portas para viabilizar o acesso, mas tinha como intuito transformar o contexto educativo e oferecer oportunidades a todos a fim de aprenderem juntos. Isso demandou um movimento de mudança que favorecesse a todos e promovesse uma reflexão dirigida a cada um, com o objetivo de tornar acessível o processo de ensino-aprendizagem por meio da articulação de dispositivos de intervenção. Um percurso que discutisse a relação entre os pares e a execução das aulas, favorecendo a construção de saberes a partir dos conhecimentos e das possibilidades reais, considerando os pontos fortes do território, assim como a diversidade das regiões da Itália e de um sistema de educação centralizado do estado (MPI, 1982; DE ANNA, 1983; 1998; 1999; 2014a; 2018; MACCIONI; ROSSI, 2018). Mais recentemente, o governo italiano se dedicou à aplicação da ideia de inclusão, implementando dispositivos normativos mais recentes, como a Lei 107-2015 sobre a Boa escola do Governo Renzi, e a revisão do Decreto Legislativo 66-2017, relativo à aplicação da Lei de 2015, aprovada pelo Governo Conte (2019-2020).

A função da formação na mudança das representações da deficiência e na mudança de olhar sobre as necessidades educativas especiais é crucial para promover modificações nas práticas educativas quando os professores não reconhecem a heterogeneidade dos alunos e quando não adequam seu ensino de modo a torná-lo acessível às diferentes necessidades e estilos de aprendizagem. Com efeito, é frequente que vários professores, em particular do 
ensino médio, apliquem as didáticas disciplinares de sua área, e considerem que cabe apenas ao professor especializado trabalhar as técnicas de adaptação às dificuldades e deficiências, sem considerar a Lei 104-1992 sobre a colaboração do professor especializado em sala de aula. Segundo a Lei, o professor especializado é corresponsável pela avaliação de todos os alunos da classe.

Nos processos que realizamos na Itália sempre abordamos esta colaboração por considerar a especificidade do aluno como uma inspiração para os outros colegas. Além disto, no início de nossos cursos, trabalhamos com os professores para conscientizá-los de suas representações e de seu olhar em relação aos alunos com necessidades educacionais especiais. Isto permite um maior reconhecimento da heterogeneidade da sala de aula, que deve ser traduzida em um conceito pedagógico e didático acessível a todos os alunos. É a partir de uma concepção pedagógica e didática acessíveis que são estabelecidas relações equitativas, fundadas na reciprocidade, que permitem caracterizar os conteúdos escolares nos princípios de autonomia e de autodeterminação, relacionados ao projeto de vida e à realização da independência no seio da sociedade. Neste âmbito, as formas de acompanhamento e as redes de apoio podem ser implantadas a fim de serem compartilhadas com todos os atores, desenvolvendo "uma relação de ajuda e apoios de proximidade" (CANEVARO, 2013; CANEVARO; MALAGUTI,2 014) disponíveis às pessoas com necessidades especiais durante toda a sua vida. Desse modo, os contextos não se limitam a uma dimensão exclusivamente médica, mas também à educação, à cultura, ao trabalho, ao esporte, ao lazer, à sexualidade, dentre outras dimensões (UN, 2006).

Nesse sentido as representações sociais tornam-se uma ferramenta pedagógica e didática eficaz para o desenvolvimento de novos modelos de interpretação da deficiência e da diversidade (COVELLI, 2015; 2016a; 2016b). Com efeito, no âmbito das atividades educativas e formadoras, ressaltar as particularidades, a diversidade e os diferentes desafios culturais, pessoais e sociais, favorece o desenvolvimento da educação para todos, a partir de exemplos concretos de temas relacionados à deficiência e à inclusão, vivenciadas no trato com os sujeitos (DE ANNA, 1998; 2014a; 2014b).

A atenção às representações e ao reconhecimento da heterogeneidade está na base de todas as atividades que nos permitem trabalhar com a formação dos professores visando uma transformação da concepção pedagógica e didática em termos de acessibilidade. Isto significa formar para o reconhecimento da diferença e para assim poder projetar o seu valor. A sensibilização e a tomada de consciência difundidas por muitos ao se referir à educação, à formação e à produção cultural devem se traduzir em oportunidades de ampliação das numerosas facetas que determinam a complexidade das experiências ligadas à deficiência e à vulnerabilidade do ser humano (GARDOU, 2005; 2012). A simplificação das numerosas experiências humanas, assim como das situações de deficiência instituídas com a utilização de modelos estereotipados e preconceituosos, não nos permite distinguir a realidade, que não pode ser reduzida a um simples dualismo decorrente do estabelecimento de conexões frágeis e, em diversos aspectos, inconclusivas, entre o que é comum e o que é considerado fora da normalidade no convívio social. 
Concluindo, nosso discurso sobre as representações, a cultura e o desenvolvimento das sociedades inclusivas não quer ser um discurso idealista ou utópico. Abordamos um aspecto que concerne diretamente a vida cotidiana de todos, porque uma escola e uma sociedade inclusiva contribuem objetivamente para a melhoria da qualidade de vida, não apenas das pessoas em situação de deficiência, mas de todos os cidadãos. Respeitar os pares e participar ativamente da vida social permite à pessoa experimentar uma vida digna e qualitativamente significativa (DE ANNA; COVELLI, 2013). Com efeito, retomando a contribuição de Sen e Nussbaum sobre o Capabillity Approach ${ }^{2}$, podemos compreender que permitir à pessoa viver as experiências e os papeis que ela escolheu livremente dentro de um contexto de alternativas - as capacidades, segundo Sen - permite à pessoa se exprimir e funcionar no sentido descrito pela Classificação Internacional de Funcionalidade/Organização Mundial de Saúde (CIF/OMS) e não na lógica da habilidade em termos de atuação absoluta (DOVIGO, 2007).

Portanto, para que a sociedade seja efetivamente inclusiva, ela deve garantir à pessoa a possibilidade de fazer escolhas dentre diferentes alternativas a fim de que suas necessidades não sejam mais vistas em termos deficitários, como falhas e inabilidades, mas como condição antológica de cada pessoa. Do mesmo modo, é preciso que as pessoas se encontrem em condição de participar da vida social (FOUGEYROLLAS, 2010; MURA 2011).

\section{Apontamentos da pesquisa sobre representações, motivações e desenvolvimento das práticas para a acessibilidade no contexto escolar}

No âmbito da formação inicial e em serviço dos professores, a abordagem metodológica da pesquisa foi estruturada por meio da criação de grupos de discussão com os professores em formação afim de analisar os aspectos culturais, estruturais, pedagógicos e didáticos que entravam ou facilitam a acessibilidade do ambiente escolar, interferindo em sua dimensão inclusiva.

Na primeira fase, o trabalho desenvolvido com 48 grupos de discussão, composto de 240 docentes em formação, permitiu a cada grupo estabelecer um registro dos aspectos mais significativos que se encontram na base da qualidade da educação inclusiva. Os registros foram analisados considerando as proposições expressas e sua frequência, o que permitiu destacar os elementos considerados mais significativos para os professores. Diante disso, foram detectados cinco macroindicadores para o aprimoramento da qualidade da educação segundo o olhar dos professores, como exposto abaixo:

1. Assegurar a plena realização e o bem-estar do aluno como pessoa em todos os aspectos (sociabilidade, comunicação, dimensões cognitivas e emocional).

2. Individualizar, personalizar e planejar atividades inclusivas intra e extraescolares para o êxito da formação.

3. Favorecer as redes de sinergia dos diferentes atores educativos, sociais e da saúde junto à família para a realização da integração e da inclusão (corresponsabilidade 
pela ação educativa, troca de informações, autorreflexão e capacidade para interrogar-se).

4. Formar e manter em processo de formação permanente e em serviço os docentes das disciplinas específicas e os professores especializados por meio de cursos produzidos dentro e fora da escola, como seminários e conferências.

5. Acompanhar permanentemente a qualidade das estruturas e dos serviços.

A análise dos relatórios dos grupos de discussão mostra o nível de densidade semântica que os professores focalizaram em relação aos alunos com NEE e, ao mesmo tempo, no trabalho efetuado na sala de aula para favorecer a auto realização e o bem-estar de todos os alunos (indicador 1:52\% da frequência toda). Segundo os professores, este aspecto coincide com as finalidades de uma escola acessível e inclusiva e deve-se sustentar em um conceito individualizado e personalizado das atividades escolares (Indicador 2:26\%). Isso permite implicar todos os alunos no cumprimento de seus deveres, em atividades que envolvem tarefas individuais e/ou coletivas, na organização de atividades comuns que permitam construir ferramentas acessíveis para todos, com a colaboração de professores das diferentes disciplinas que possam lidar com as diversas formas de aprender. Esta concepção implica estabelecer interações e sinergias com vários atores, colegas, gestores, assistentes, etc. (Indicador 3:15\%). As propostas relativas à formação em serviço do professor (Indicador 4:4\%) e o monitoramento da qualidade da inclusão escolar (3\%) obtiveram baixas porcentagens (COVELLI, 2016b; DE ANNA 2016a) na escala de prioridades relativas à acessibilidade de uma escola em vias de se tornar uma escola inclusiva.

No que diz respeito às motivações dos professores em relação à questão do apoio, nós observamos uma transformação pessoal. As repostas coletadas no início dos cursos demonstravam um sentimento marcado pelo medo e, ao mesmo tempo, pelo interesse e curiosidade em relação à deficiência e às pessoas que vivenciam esta situação, como demonstra o gráfico 1:

Gráfico 1: Sentimentos dos professores em relação às pessoas em situação de deficiência antes da formação

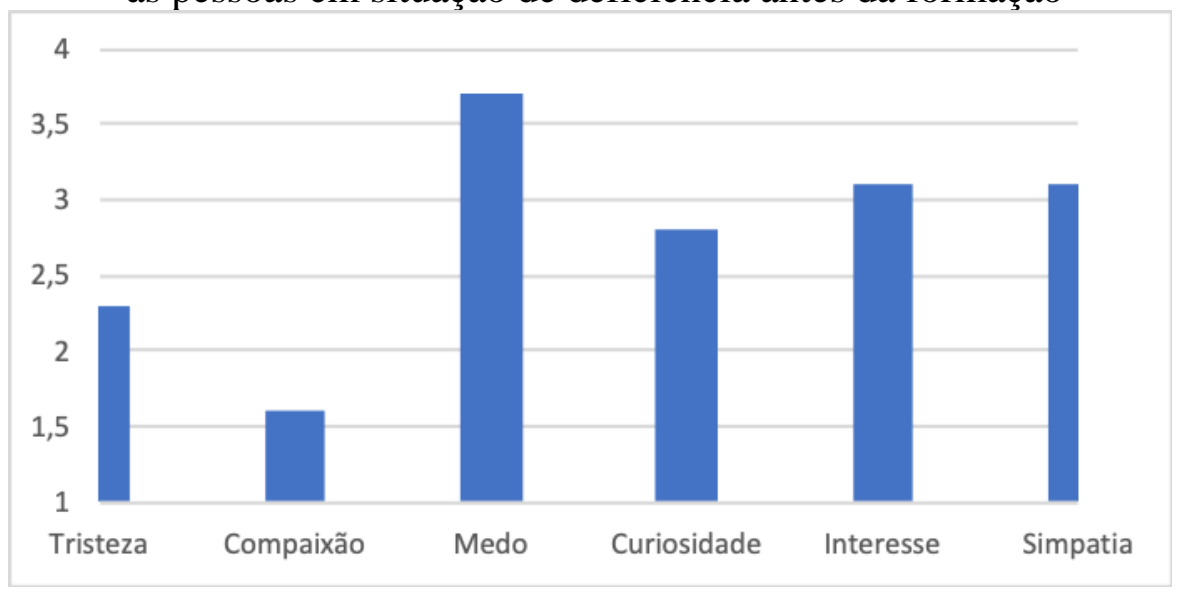


A formação teve um papel essencial na mudança de olhar dos professores nesse sentido, pois após conhecerem uma pessoa em situação de deficiência e realizarem as atividades de formação, os docentes relataram sentirem-se mais abertos e tolerantes, mais ativos, sensíveis e com uma maior abertura, interesse e simpatia. O medo, frequentemente acompanhado por um sentimento de inadequação profissional (COVELLI, 2015), reduziuse graças às possibilidades de conhecimento e desenvolvimento das competências oferecidas pelo Curso de especialização em apoio (DE ANNA, 2014c)

É possível verificar no Gráfico 2 que os professores cursistas adquiriram uma melhor aptidão relacional e comunicacional, assim como um maior conhecimento sobre as discussões específicas. Além disso, os temores iniciais de não poderem interagir com os estudantes e em relação à intervenção foram atenuados, e os professores discutiram entre eles atividades visando promover a aprendizagem. Com os conhecimentos trabalhados, o compartilhamento de experiências apresentadas pelos formadores e por meio dos estágios nas escolas, houve mudança do olhar dos professores e eles compreenderam melhor como abordar os problemas e procurar soluções possíveis.

Gráfico 2: Mudança de olhar dos professores diante das pessoas em situação de deficiência após a formação

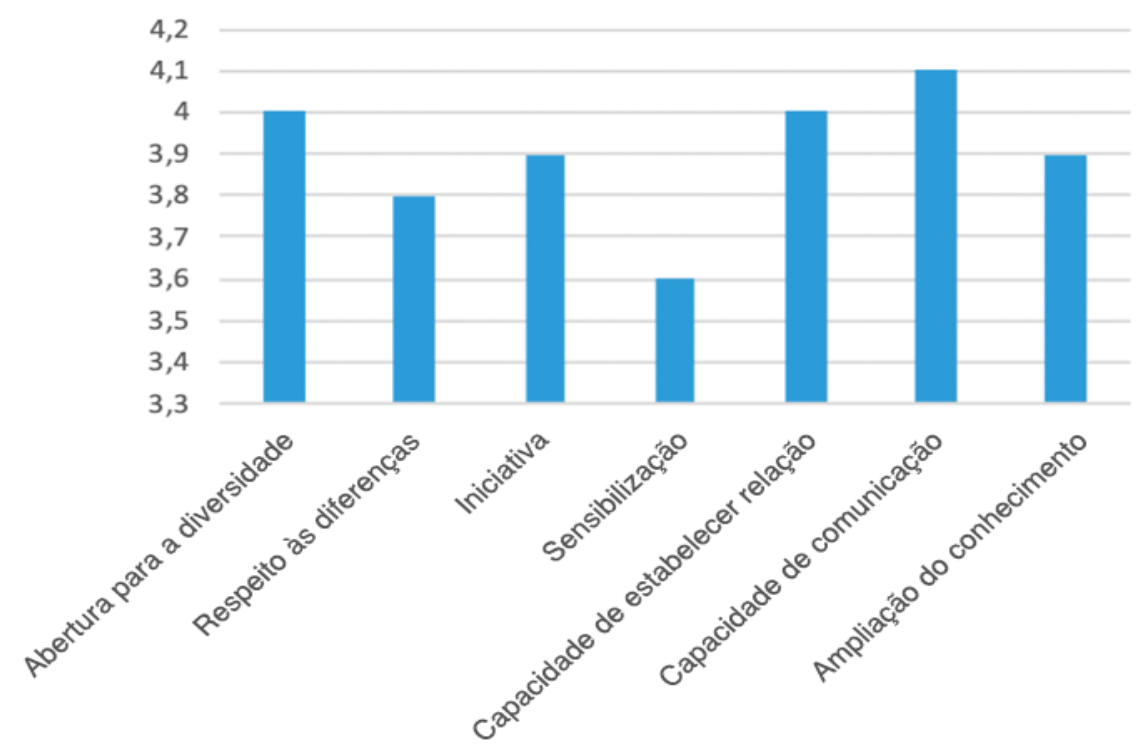

Após a formação e a realização das atividades de estágio nas escolas, os professores superaram vários preconceitos. As oportunidades de conhecer e trabalhar com alunos em situação de deficiência reduziu o sentimento de medo, permitindo uma maior abertura para a diversidade, bem como um aprimoramento das condições relacionais e comunicacionais A mudança de olhar dos professores ocorreu a partir do entendimento de que o aluno em situação de deficiência é uma referência para toda a sala de aula, e que os mesmos não 
significam um entrave para a aprendizagem dos outros estudantes. Esses mesmos professores ressaltaram os seguintes aspectos, indispensáveis para um ambiente escolar acessível e inclusivo:

1. Na gestão e no desenvolvimento dos processos de integração e de inclusão para todos os alunos: a) apoiar o percurso de socialização, aprendizagem e escolarização do aluno em situação de deficiência junto aos seus colegas; b) observar, visando a criação de uma relação de empatia e confiança; c) promover uma sensibilização cultural; d) transformar as relações entre o aluno com NEE e o contexto da escola; conhecer a vivencia do aluno com NEE; e) apoiar e interpretar as necessidades educativas do aluno com NEE.

2. Na mediação e partilha do percurso educativo do aluno com NEE: a) adotar estratégias de cooperação e buscar uma sinergia entre os professores de área e os professores de apoio; b) implicar os alunos, os trabalhadores da escola, as famílias, os profissionais dos serviços de apoio e da área da saúde, assim como as associações locais, de forma a obter um maior apoio no Conselho de classe para a adoção de projetos inclusivos.

3. Na elaboração de estratégias educativo-didáticas e na preparação de diferentes situações que priorizem a aprendizagem para toda a sala de aula, bem como a realização do projeto de vida: a) facilitar a aprendizagem e a comunicação; b) valorizar os potencias e o desenvolvimento da subjetividade de todos os alunos e não somente do aluno com NEE.

Ao final do percurso de formação, no âmbito de defesa do exame para obtenção da certificação, professores em formação apresentaram um relatório final das atividades de estágio realizadas na escola (com o acompanhamento de um tutor da escola e outro da universidade); um produto multimídia sobre a aplicação das Tecnologias da Informação e da Comunicação (TIC) nas atividades didáticas; aprofundamentos teóricos e práticos relativos à disciplinas do curso.

A leitura das diferentes experiências desenvolvidas nas salas de aula heterogêneas permitiu confrontar a aplicação dos princípios expostos na realidade escolar com a programação das iniciativas de pesquisa-ação em diferentes momentos do percurso de escolarização (maternal, primário e ensino médio). Um exemplo disso foi a utilização didática da pesquisa como expressão das emoções; a aprendizagem da matemática por meio do corpo e da motricidade (DE ANNA, 2009); a narração da aprendizagem da leitura e da literatura utilizando as Cartas de Propp e a transformação de Rodari ${ }^{3}$ (DE ANNA; ROSSI; MAZZER, 2018); as atividades didáticas escolares e extraescolares de teatro (VILLANELLA, 2013); a utilização das tecnologias na formação inclusiva e a aplicação do Desenho Universal de Aprendizagem (CAST, 2011; DE ANNA 2014a; SANCHEZ, 2016; PAGLIARA; SANCHEZ; DE ANNA, 2017), dentre outros.

Os professores compreenderam que trabalhar de forma interdisciplinar, por meio de experiências criativas e inovadoras, proporciona uma oportunidade para todos e que em um ambiente adaptado é possível valorizar a especificidade de cada um, assim como adequar as 
ferramentas necessárias para melhorar a aprendizagem e o acesso aos conteúdos disciplinares.

\section{A importância da colaboração nos processos inclusivos}

As atividades de pesquisa relativas aos processos de inclusão escolar ocorreram nos anos seguintes com o propósito de analisar, de maneira mais analítica, a avaliação da qualidade das escolas das regiões de Lácio e Campânia. Nessa segunda fase, durante os cursos de formação em serviço, a perspectiva das representações foi utilizada nas análises dos professores-coordenadores sobre a inclusão em suas escolas. Da mesma forma, em um primeiro momento, no âmbito da pesquisa-ação, criamos grupos de discussão com os docentes para realizar atividades que simulavam avaliação do processo de inclusão em suas próprias escolas. A análise do conteúdo permitiu isolar os descritores mais significativos em termos de frequência, que foram incluídos em um questionário semiestruturado, composto de 65 itens e organizadas a partir de três macro dimensões de análise: Cultura inclusiva e formação dos profissionais da escola; Gerenciamento, organização escolar e colaboração entre os atores implicados no processo de inclusão; Concepção pedagógica e didática.

Os itens foram avaliados pelos professores por meio de uma escala Likert de 1 (insuficiente) a 5 (excelente), e o questionário foi submetido a 291 docentes, dentre os quais 192 já eram especializados no apoio e 99 encontravam-se em processo de especialização no mesmo tema. Tratavam-se, portanto, de duas amostras diferentes em termos de experiência profissional. A comparação das médias entre as duas indica que estas diferenças estão ligadas, principalmente, às particularidades territoriais das regiões pertencentes aos grupos de Lácio e Campânia, e não ao tipo de formação. Em relação aos professores que se encontravam em processo de especialização no apoio, tratava-se de profissionais qualificados para ensinar os conteúdos disciplinares e que já conheciam os contextos escolares. Entretanto, o ponto mais crítico que surgiu das avaliações, tanto de Lácio, quanto de Campânia, refere-se à dimensão colaborativa, em específico, no que concerne as relações entre escola e serviços locais de saúde. Nesse sentido, enquanto a avaliação da qualidade da inclusão escolar era de 3,67, a avaliação da sinergia entre a escola e os profissionais da saúde para a realização da inclusão obteve uma média de 3,21.

As dificuldades de colaboração entre as escolas e os serviços territoriais representam um problema cultural que tem fortes repercussões sobre a qualidade geral dos processos inclusivos nas escolas. Para ilustrar para o leitor que não conhece bem a realidade italiana as dimensões dessa questão, é necessário explicar que desde o início do processo de integração e de inclusão, a Lei 517/1977 demandava uma colaboração integrada entre escola, serviços sociais e saúde, bem como a colaboração das famílias e das associações dos pais das pessoas em situação de deficiência. Desde o início de nossas experiências de inclusão escolar essa sinergia institucional foi considerada um fator estratégico no desenvolvimento destes processos. Para tanto, foram criados dispositivos colaborativos e acordos locais e nacionais entre os Ministérios da Educação, Saúde e Assistência social 
(Lei 104 /92, de 24/02/1994; Lei 328, de 2000 e Lei 107 de 2015). Essa articulação visava organizar atividades em comum e sustentar o processo de inclusão das pessoas em situação de deficiência (NEE), com a finalidade de construir um projeto de vida para essa população (Conferência do Estado e regiões, de 2008; Diretrizes de base, Miur, 2009; Reforma Gelmini, 2009/2010; Acordos entre Ministérios da Educação e da Saúde, 2012). Esse modelo de organização institucional foi analisado também no projeto OCDE/CERI, denominado "A inserção escolar das pessoas com deficiência: estabelecimentos para todos" (OECD, 1999), que estabelece práticas de integração para os diferentes países da OCDE e distingue três fases para a estruturação de unidades de referência em cada país: Descrição e funcionamento da unidade; Continuidade, êxito das intervenções, participação da escola (professores, alunos, pais e gestores); Supressão dos obstáculos encontrados, problemas econômicos e outras informações úteis para uma avaliação eficaz. O objetivo deste programa era demonstrar a implementação da integração no meio escolar, definida pelos estados membros da OCDE com aquela que apresenta o "máximo de interação entre pessoas deficientes e não deficientes" no contexto comum. Isto levou a Itália (que havia participado de todas as mesas de discussão em Paris e, também, de conferências em diversos países, notadamente nos Estados Unidos) a difundir trabalhos realizados em unidades de referência, escolhidas nas regiões da Toscana, Lácio, Basilicata, Úmbria (DE ANNA, 1992a; 1992b; 1998). Os 18 professores que trabalharam no projeto italiano tiveram a oportunidade de realizar encontros e visitas a escolas francesas situadas em Paris e na periferia, e nos institutos de formação de Beaumont e de Suresnes. Tendo como base indicadores da OCDE foram produzidos: Protocolos de acordo; -relatórios médicos; programação conjunto entre conselhos institucionais e profissionais para a integração; trabalho com Conselho de classe; perfil dinâmico-funcional; programação didáticaeducativa (curriculum instrumental e curriculum funcional); metodologias práticas; e verificações. No tratamento dos pontos específicos, as diferentes questões foram explicadas a fim de destacar as tarefas e as implementações, as responsabilidades, a frequência das reuniões e das intervenções, o trabalho conjunto com os professores das disciplinas e os professores de apoio (de Anna, 1992b).

No decorrer da terceira fase do projeto, os representantes da OCDE se reuniram (12 a 15 de abril/1997) na Universidade de Siena por ocasião da conferência "Universidades e necessidades educativas especiais", com o patrocínio da Fundação Europeia de Ciência, do Ministério da Universidade, Ciência e Tecnologia (MUST) e da OCDE. Na continuidade das fases descritas acima, a OCDE, em colaboração com a Universidade de Roma, administrações locais, escolas e com a Superintendência escolar regional do Lácio, organizaram visitas guiadas e escolheram uma região de referência no território. Essa região deveria desenvolver um trabalho articulando as intervenções escolares e promovendo uma colaboração entre o serviço social, a saúde, as atividades do Centro de Formação Profissional e a Universidade. (OCDE, 1999, relatório sobre a Itália, p.205-232).

No seio da OCDE, o estudo dos indicadores do processo de integração em um contexto internacional destacou as dificuldades de se estabelecer comparações tendo em vista a heterogeneidade dos sistemas educativos, das organizações, dos papéis exercidos pelos 
profissionais, das colaborações desenvolvidas no território, que colocavam em jogo diferentes responsabilidades na análise do contexto, nas visitas guiadas e nas pesquisas feitas com o objetivo de compreender melhor as situações reais. Nesses anos, na Itália, em particular no Lácio, já eram visíveis as colaborações ativas existentes entre as autoridades locais, os serviços sociais e de saúde com as escolas, que produziam reflexões importantes e conhecimentos compartilhados por diferentes profissionais, influenciando não apenas as práticas pedagógicas, mas, também, as relações com as famílias em torno da construção de um projeto de vida.

Na Itália, o estudo dos indicadores foi retomado em seguida no âmbito de um intensivo trabalho do Observatório do Ministério da Educação nos anos de 1996 a 2000, fomentando as competências dos professores das disciplinas e dos professores de apoio por meio da formulação de sete módulos de formação e com a aplicação de temáticas relacionadas aos cursos de especialização em serviço para os professores (Circular Ministério da Educação, de 20 de Dezembro de 1999). Também foram criados projetos ativos de pesquisa e de formação sobre a utilização das tecnologias, formação profissional e estratégias pedagógicas integradas nos processos de inclusão (DE ANNA, 2007; 2008; 2014a).

O trabalho e o compromisso de divulgação de boas práticas pela Editora Erickson, sustentado pelo Comitê cientifico de Pedagogia Especial, que é coordenado por Andrea Canevaro (Universidade de Bolonha) também foi significativo nesse contexto, divulgando publicações cientificas, promovendo conferências bienais e atividades de formação mais especificas (IANES; TORTELLO, 1999; CANEVARO; D'ALONZO; LANES, 2009; IANES; CANEVARO, 2008; D'ALONZO; CALDIN, 2012).

\section{Conclusões}

As análises efetuadas nos diferentes ciclos do curso de especialização para os professores de apoio e nos cursos de formação em serviço que tiveram como referência a inclusão nas escolas do Lácio e Campânia permitem representar, de forma analítica, as múltiplas dimensões subjacentes aos processos de inclusão. Todavia, o tratamento dos dados quantitativos detectou alguns problemas críticos da formação continuada, da formação dos professores e do pessoal auxiliar. Além disso, segundo os professores, para ampliar a qualidade da inclusão escolar na Itália, seria necessário: aumentar o número dos profissionais qualificados; estabelecer um maior compartilhamento das informações sobre a transição dos alunos para outros níveis escolares; favorecer uma maior colaboração entre os professores das áreas disciplinares e os professores especializados no projeto educativo e pedagógico da sala de aula; ampliar a colaboração com os governos locais e as famílias; e criar um acompanhamento da qualidade das estruturas e dos serviços ${ }^{4}$.

Desde as primeiras pesquisas até as atuais, podemos constatar que um dos aspectos mais desafiadores desse processo ainda é efetivar a colaboração entre as escolas, as autoridades locais, os serviços sociais e de saúde, bem como envolver as famílias em uma parceria compartilhada e responsável. No âmago das escolas italianas, também ficou 
determinada a criação de uma coordenação de apoio para assegurar o processo de inclusão e incentivar os serviços de cada região a serem mais presentes nessa parceria. Esse ponto emergiu a partir das crises econômicas vivenciadas pela Itália nesse período (DE ANNA; COVELLI; MAZZER, 2019). Na pesquisa da OCDE, percebemos um maior empenho do Ministério da Educação na análise das situações territoriais afim de promover suportes econômicos para as diferentes regiões no que diz respeito à educação inclusiva. Atualmente, a discussão está aberta entre as regiões e o Estado para descentralizar a responsabilidade pela educação.

Nos grupos de pesquisa, tentamos documentar as boas práticas utilizando de métodos narrativos e descritivos (DE ANNA 1992a; 1992b; 1996; 1998; 2003; 2013; DE ANNA; ROSSI; MAZZER, 2018). No doutorado internacional Culturas, deficiência, inclusão: educação e formação, da Universidade de Roma, foro itálico, trabalhamos com os estudos relacionados à percepção dos professores e suas práticas (TINE, 2012) e com pesquisas sobre as famílias (DIALLO, 2012), que abordam as motivações e competências dos professores (DE ANNA; GARDOU; COVELLI, 2018; FUMES; SANTOS; DAMATO, 2018), para observar a aplicação e a confrontação do Desenho Universal de Aprendizagem em conexão com as práticas e estratégias didáticas dos professores na Itália (SANCHEZ; MAZZER; PAGLIARA; DE ANNA, 2017; SANCHEZ; DE ANNA; MAZZER; PAGLIARA, 2019).

Como conclusão, consideramos a aplicação do Desenho Universal de Aprendizagem sobre as práticas educativas italianas, por meio de várias pesquisas em curso, para demonstrar que a Itália é pioneira da integração e da inclusão escolar (CARAGLIO; GAVINI 2018;), tendo construído, durante 42 anos de experiência, várias estratégias didáticas para o trabalho em sala de aula, visando tornar a aprendizagem acessível e flexível para todos (GHEDIN; MAZZOCUT, 2017; SANCHEZ; DE ANNA; MAZZER; PAGLIARA, 2019).

Em 2019, tínhamos 450 estudantes em formação na especialização de apoio, sendo 300 na Universidade de Roma foro itálico e 150 na Universidade Tor Vergata, também de Roma. Nós estamos analisando, ainda na situação atual, como a formação e a pesquisa podem contribuir para modificar o olhar e construir contextos inclusivos por meio da transformação das competências educativas e didáticas para a inovação e acessibilidade nos ambientes escolares.

\section{Notas}

1. As oito competências-chaves são: Comunicação na língua materna; Comunicação em uma língua estrangeira; Competência matemática, científica e tecnológica; Competência digital; Aprender a aprender; Competências sociais e cívicas; Espírito de iniciativa e espírito empresarial; Sensibilidade e expressão culturais (Nota da Tradutora).

2. Para aprofundamento, indicamos o livro NUSSBAUM, Martha; SEN, Amartya (Ed.). The quality of life. Oxford: Clarendon, 1993 (Nota da tradutora).

3. Inspirado na obra Morfologia do Conto Maravilhoso (1928), do russo Vladimir Propp (1895-1970), o escritor italiano Gianni Rodari (1920-1980) elaborou um jogo de cartas para inspirar a construção de histórias. Em 1973, Rodari escreveu o livro Gramática da Fantasia onde aborda esse tema. Lucia De Anna e Marzia Mazzer (2018) produziram 
um artigo sobre a narrativa e a experiência de um professor que utilizava as Cartas de propp de maneira inclusiva, em uma classe onde estudava um aluno cego.

4. Esta contribuição parcial de Lucia de Anna sobre as atividades de pesquisa faz parte de uma exposição comparativa mais ampla, realizada em colaboração com outros países, e que foi apresentada em conferência realizada na cidade de Fortaleza (Ceará), por ocasião do Colóquio Internacional sobre a Diversidade e Inclusão (30 de janeiro a 3 de fevereiro de 2018), sob coordenação de Eric Plaisance. O material apresentado está em fase de publicação e será divulgado em francês e em português. A análise foi apresentada, ainda, em Conferência realizada em Lille (julho de 2018). Os dados elaborados e aprofundados das pesquisas foram apresentados para publicação em um artigo na Revista ALTER, de autoria de Alessio Covelli e Lucia de Anna.

\section{Referências}

AINSCOW, M. Dans L’éducation inclusive : une formation à inventer, Unesco. UNESCO, Paris, 2014, p. 115-120.

CANEVARO, A. Scuola inclusiva e mondo più giusto. Erickson, Trento, 2013.

CANEVARO, A., D'ALONZO, L. et IANES, D. (dir.). L'integrazione scolastica di alunni con disabilità dal 1977 al 2007. Risultati di una ricerca attraverso lo sguardo delle persone con disabilità e delle loro famiglie. Bozen-Bolzano University Press, Bolzano, 2009.

CANEVARO, A. et MALAGUTI, E. Inclusione e Educazione: sfide contemporanee nel dibattito intorno alla Pedagogia speciale. Italian Journal of Special education for Inclusion, 2(2), p. 97-108, 2014.

CARAGLIO, M. et GAVINI, C. L'inclusion des élèves en situation de handicap en Italie. Rapport I.G.A.E.N.R. - Février 2018 [En ligne]. Disponible à l'adresse: https://www.education.gouv.fr/cid127533/ 1-inclusion-des-eleves-en-situation-de-handicap-en-italie.html

CAST. Universal Design for Learning (UDL) Guidelines version 2.0. Author, Wakefield. Trad. it. version 2.0, de Savia, G et Mulè, P. [en ligne], 2011. Disponible à l'adresse : http://www.udlcenter.org/sites/ udlcenter.org/files/UDL\%20Linee\%20guida\%20Versione\%202.0\%20ITA.doc.

COVELLI, A. Verso una cultura dell'inclusione. Rappresentazioni mediali della disabilità. Aracne, Roma, $2016 a$.

COVELLI, A. Inclusion Quality Indicators in the Training of Teachers. In: Teaching Accessibility and Inclusion, DE ANNA, L., Carocci, Roma, 2016b, p. 130-142.

COVELLI, A. Le rappresentazioni sociali come strumento didattico pedagogico di inclusione. In: L'insegnante specializzato. Itinerari di formazione per la professione, DE ANNA, L.; GASPARI, P.; MURA, A. (dir.), FrancoAngeli, Milano, 2015, p. 137-150.

D’ALONZO L. et CALDIN, R. (dir.). Questioni, sfide e prospettive della pedagogia speciale. Liguori, Napoli, 2012.

DE ANNA, L. Aspetti normativi dell'inserimento sociale degli handicappati in Italia e all'estero. Tempinuovi, Roma, 1983.

DE ANNA, L. Il diritto allo studio. La sentenza della Corte Costituzionale 215/87. Aspetti pedagogici, didattici e giuridici. L'ED, Roma, 1992a.

DE ANNA, L. Integrazione scolastica. Modelli operativi e sistemi a confronto. L'ED, Roma, 1992 b.

DE ANNA, L. Pedagogia speciale. Guerini, Milano, 1998.

DE ANNA, L. Le politiche integrative dell'educazione scolastica dei disabili 1996-1999. Modulo Europeo. Università Roma Tre, Roma, 26441-MG2-1-96-IT ERASMUS - EEM, 1999.

DE ANNA L. Diversité et handicap à l'école. In: BELMONT B.; VERILLON A.. Quelles pratiques éducatives pour Tous?. p. 37-59, Paris:CTNERHI E INRP, 2003. 
DE ANNA, L. La formation des enseignants: le processus d'intégration en Italie. Dans Conférence Le nouvelle revue de l'AIS - Adaptation et intégration scolaire 1954-2004, 50 ans de formations spécialisées, AIS, Paris, 2004.

DE ANNA, L. La formazione degli insegnanti e i sette Moduli. L'Integrazione scolastica e sociale, 6(5), p. 437-453, 2007.

DE ANNA, L. Les processus d'intégration scolaire en Italie et la formation des enseignants. Reliance, 27, p. 111-120, 2008.

DE ANNA, L. Formation en Italie des enseignants accueillant des enfants Handicapés. Recherche et Formation, v. 61, p. 55-70, 2009.

DE ANNA, L. L'identità della persona con disabilità nella Convenzione ONU del 2006. Evoluzione storica attraverso i documenti internazionali. In: Pedagogia speciale oltre la scuola. Dimensioni emergenti nel processo di integrazione, MURA, A. (dir.), FrancoAngeli, Milano, 2011, p. 23-39.

DE ANNA, L. La pedagogia speciale in Europa. I processi di integrazione e inclusione nel confronto con altri paesi europei ed extraeuropei. In: Questioni, sfide e prospettive della pedagogia speciale, D'ALONZO, L.; CALDIN, R. (dir.), Liguori, Napoli, 2012, p. 41-60.

de Anna, L. A educação especial, a pesquisa, a formação e a inclusão escolar na Itália. In: SANTOS, B. S.; DE ANNA, L. (orgs), Espaços psicopedagógicos em diferentes cenários, Porto Alegre: EDIPUCRS, 2013, p. 190-208.

DE ANNA, L. Pedagogia Speciale. Integrazione e Inclusione. Carocci, Roma, 2014a.

DE ANNA, L. La scuola inclusiva: ruoli e figure professionali. Italian Journal of Special education for Inclusion, 2(2), p. 109-128, 2014b.

DE ANNA, L. Enseignants et éducation spécialisée en Italie. Formation, rôle, compétences dans les contextes d'intégration et d'inclusion. La nouvelle revue de l'adaptation et de la scolarisation, 66 (2e trimestre), p. 191-205, 2014c.

DE ANNA, L. Teaching Accessibility and Inclusion. Carocci, Roma. 2016a.

DE ANNA, L. Le esperienze di integrazione e inclusione nelle Università tra passato e presente, Franco Angeli, Milano, 2016b.

DE ANNA, L. A Inclusão entre o passado e o presente: formação de professores para a educação inclusiva na Itália. In: Perspectivas Internacionais da Educação Inclusiva, Gonzales Mendes, E., Almeida, M.A. et Santos Amançio Cabral, L. (dir.), ABPEE, Marília, 2018, p. 133-150.

DE ANNA, L.; COVELLI, A. Disabilità e identità nella rappresentazione dei media. In: Identità, Soggettività e Disabilità. Processi di emancipazione individuale e sociale, MURA, A. ET ZURRU, A.L. (dir.), FrancoAngeli, Milano, 2013, p. 85-104.

DE ANNA L.; COVELLI, A.; MAZZER, M. Former à la collaboration sur l'inclusion. Rapport au Colloque International "Construire un avenir pour chaque élève", 24-25, April. Lausanne, HEP Vaud, 2019.

DE ANNA, L., GARDOU, C.; COVELLI, A. (dir.). Inclusione, culture e disabilità. La ricerca della pedagogia speciale tra internazionalizzazione e interdisciplinarità: uno sguardo ai cinque continenti. Erickson, Trento, 2018.

DE ANNA, L.; GASPARI, P.; MURA, A. (dir.). L'insegnante specializzato. Itinerari di formazione per la professione. Milano: FrancoAngeli, 2015.

DE ANNA, L.; ROSSI, C.; MAZZER, M. Inclusione, narrazione e disturbi dello spettro autistico. Ricerche e prospettive della pedagogia speciale. Cafagna, Barletta, 2018.

DIALLO, K. Identité et inclusion sociale. Le handicap, enjeu des interactions sociales : le cas des élèves des écoles secondaires supérieures de Rome et Cagliari en Italie. Thése du Doctorat International «Culture, disabilità, inclusione: educazione e formazione»(XXIV cycle). Università degli Studi di Roma "Foro 
Italico", Roma, 2012.

DOVIGO, F. Fare differenze. Indicatori per l'inclusione scolastica degli alunni con bisogni educativi speciali. Erickson, Trento, 2007.

EBERSOLD, S. Transition juvénile, accessibilité et citoyenneté. Dans L. DE ANNA; GARDOU, C.; COVELLI, A (dir.), Inclusione, culture e disabilità. La ricerca della pedagogia speciale tra internazionalizzazione e interdisciplinarità: uno sguardo ai cinque continenti. Erickson, Trento, 2018, p. 130-149.

FERRUCCI, F. La disabilità come relazione sociale. Gli approcci sociologici tra natura e cultura. Rubbettino, Catanzaro, 2004.

FOUGEYROLLAS, P. Le funambule, le fil et la toile. Transformations réciproques du sens du handicap. Les Presses de l'Université Laval, Quebec, 2010.

FREIRE, P. Pedagogia dell'autonomia. Saperi necessari per la pratica educativa. EGA, Torino, 2004. Versão em português:

FREIRE, P. Pedagogia da autonomia. São Paulo: Paz e Terra, 2019.

FREIRE, P. L'educazione come pratica della libertà. Mondadori, Milano, 1973. Versão em português:

FREIRE, P. Educação como prática da liverdade. São Paulo: Paz e Terra, 2019.

FUMES N.L.F.; SANTOS, S.D.G.; DAMATO T.A.L. (dir.). Possibilidades e Desafios para a Inclusão Escolar no Campo da Educação Especial. EDUFAL: Imprensa Oficial Graciliano Ramos, Maceió, 2018.

GARDOU, C. Fragments sur le handicap et la vulnérabilité. Erès, Toulouse, 2005.

GARDOU, C. La société inclusive, parlons-en ! Erès, Toulouse, 2012. Versão em português:

GARDOU, C. A sociedade inclusiva: Falemos dela! Não há vida minúscula. Belo Horizonte: Editora Fino Traço/UFMG, 2018.

GHEDIN, E; MAZZOCUT, S. Universal Design for Learning per una valorizzazione delle differenze: un'indagine esplorativa sulle percezioni degli insegnanti. Giornale Italiano della Ricerca Educativa Italian Journal of Educational Research, 10(18), p. 145-162, 2017.

HONNETH, A. Lotta per il riconoscimento. La grammatica morale dei conflitti sociali. Il Saggiatore, Milano, 2002.

IANES, D. La speciale normalità. Erickson, Trento, 2006. Versão em português: IANES, D. A especial normalidade. São José dos Campos (SP): Pulso editorial, 2010.

IANES, D.; CANEVARO, A. (dir.). L'integrazione scolastica. Tendenze, strategie operative e 100 buone prassi. Erickson, Trento, 2008.

IANES, D.; TORTELLO, M. (dir.). La Qualità dell'integrazione scolastica. Erickson, Trento, 1999.

MACCIONI, I.; ROSSI, C. La Legge dell'Inclusione nella Scuola italiana. A quarant'anni dalla 517/77. Albatrello, Arezzo, 2018.

MOLITERNI, P. Didattica e scienze motorie. Tra mediatori e integrazione. Armando, Roma, 2013.

MPI. Seminario Nazionale di Arezzo: Iniziative pedagogico-didattiche per l'inserimento scolastico degli handicappati. Nuova Stampa, Città di Castello, 1982.

MURA, A. L'accessibilità. Considerazioni teoriche e istanze operative. In: Pedagogia speciale oltre la scuola. Dimensioni emergenti nel processo di integrazione, MURA, A. (dir.), FrancoAngeli, Milano, 2011, p. 40-60.

OECD. L'insertion scolaire des handicapés: des établissements pour tous. OECD Publishing, Paris, 1999.

OECD. TALIS 2018 Results (Volume I): Teachers and School Leaders as Lifelong Learners. OECD Publishing, Paris, 2019. 
PAGLIARA S.; SANCHEZ, U. M.; DE ANNA L. ICT and UD: Preliminary Study for Recommendations to Design Accessible University Courses. Dans P. Cudd and L. de Witte, Harnessing the Power of Technology to Improve Lives. Eds. IOS Press, Amsterdam, 2017, p. 989-992.

REMOTTI, F. L'ossessione identitaria. Rivista Italiana di Gruppo analisi, 25(1), p. 9-29, 2011.

SANCHEZ, U. M. Accessibilità pedagogica e inclusione: significati, norme e formazione con particolare riguardo alla Spagna. Thése du Doctorat International Culture, disabilità, inclusione: educazione e formazione (XXVII cycle). Università degli Studi di Roma "Foro Italico", Roma, 2016.

SANCHEZ, U. M.; MAZZER, M.; PAGLIARA, S.; DE ANNA, L. La formazione degli insegnanti di sostegno sulle TIC. Analisi dei prodotti multimediali del corso di specializzazione per le attività di sostegno. Italian Journal of Special education for Inclusion, 5(1), p. 133 -146, 2017.

SANCHEZ U. M.; DE ANNA L.,; MAZZER M.; PAGLIARA S. Les pratiques éducatives des enseignants : application de la CUA (conception universelle de l'apprentissage). Relazione al Colloquio Internazionale "Construire un avenir pour chaque élève" 24- 25 aprile 2019, HEP Vaud Lausanne, 2019.

SCHOVANEC, J. Nos Intelligences multiples: Le bonheur d'être différent. Humensis, Paris, 2018.

TINE, C.T. A la recherche de significations dans le discours et les pratiques des enseignants. Contenus et dynamique des représentations sociales de l'inclusion scolaire des enfants et adolescents en situation de handicap en Italie. These du Doctorat International «Culture, disabilità, inclusione: educazione e formazione» (XXIV cycle). Università degli Studi di Roma "Foro Italico", Roma, 2012.

UNESCO (2017). Un guide pour assurer l'inclusion et l'équité dans l'éducation [en ligne]. UNESCO, 2017, Paris. Disponible à l'adresse: https://unesdoc.unesco.org/ark:/48223/pf0000259389.

UNITED UNION. The Convention on the Rights of Persons with Disabilities, 2006.

VILLANELLA, G. The Implementation of Inclusive Education in Secondary Schools in Italy. Bulletin of Educational Resources and Research, 58 "Secondary Education in the World". National Academy for Educational Research, Taiwan, 2013, p. 71-138.

WARNOCK REPORT. Report of the Committee of Enquiry into the Education of Handicapped Children and Young People. London: Her Majesty's Stationery Office 1978. Disponível em: http://www. educationengland.org.uk/documents/warnock/warnock1978.html. Acesso em 29 dezembro 2019.

\section{Correspondência:}

Lucia De Anna: Professora e pesquisadora da Università degli Studi di Roma "Foro Italico". Atua em processos de formação de professores em parceria com várias universidades italianas e da comunidade européia.

ORCID: 0000-0002-5886-2565.

E-mail: lucia.deanna@uniroma4.it

Alessio Covelli: Pesquisador em Pedagogia, Didática especial e Pesquisa educacional da Università degli Studi di Roma "Foro Italico".

ORCID: 0000-0001-7948-2345.

E-mail: alessio.covelli@uniroma4.it

Texto publicado em Currículo sem Fronteiras com autorização dos autores. 